\title{
Existence of fractional order semianalytical results for enzyme kinetics model
}

\author{
Hussam Alrabaiah ${ }^{1,2^{*}}$ (D, Amjad Ali ${ }^{3,4}$, Fazal Haq ${ }^{4}$ and Kamal Shah ${ }^{5}$
}

\author{
*Correspondence: \\ hussam89@yahoo.com \\ ${ }^{1}$ College of Engineering, Al Ain \\ University, Al Ain, United Arab \\ Emirates \\ ${ }^{2}$ Department of Mathematics, Tafila \\ Technical University, Tafila, Jordan \\ Full list of author information is \\ available at the end of the article
}

\begin{abstract}
This research work investigates the existence of semianalytical solutions of a chemical kinematics model. We develop the conditions for the existence of the solutions for a proposed enzyme kinetics model, via tools of the fixed point theory. The semianalytical results were obtained with the help of Laplace transformation and Adomian decomposition method. The results established by the proposed techniques are in the form of infinite series. Furthermore, with extending homotopy perturbation method (HPM), we develop a series solutions for the considered model. By using Matlab, we present the approximate solution for both methods up to a few series terms.
\end{abstract}

MSC: Primary 26A33; 35A08; secondary 93A30

Keywords: Enzyme kinetics; Mathematical models; Fractional derivatives; Fixed point approach; Analytical solution

\section{Introduction}

Recently, experimental evidence shows that dynamics problems in nature follow a fractional calculus analysis. The relevant field of research is a fast growing area, due to its numerous applications in diverse and widespread fields of engineering and science; such as chemical models, physics, signal and image processing, quantum mechanics, control theory, nonlinear dynamics, biological population models, optimization theory, and much more [1-10]. Instantly, it is evident that dealing with a dynamical system with memory effects is one of the biggest challenges for researchers. Fractional calculus has a direct link to dynamical systems (with memory effect). Therefore, fractional differential equations (FDEs) present a novel technique developed to model phenomena related to the dynamics of the aforesaid fields of science [11-14]. Fractional derivatives are global in nature and offer a greater degree of freedom compared to the conventional derivatives. Numerous researchers have investigated various features of FDEs concerning the existence, stability analysis, and approximate solutions. They utilized different techniques of fixed-point theory and numerical analysis to investigate the existence theory, stability analysis, and approximate solutions of FDEs [15-18].

An important aspect of the this concerned field is mathematical modeling, which is a tool that describes almost all dynamical phenomena in mathematical language and con-

(c) The Author(s) 2020. This article is licensed under a Creative Commons Attribution 4.0 International License, which permits use sharing, adaptation, distribution and reproduction in any medium or format, as long as you give appropriate credit to the original author(s) and the source, provide a link to the Creative Commons licence, and indicate if changes were made. The images or other third party material in this article are included in the article's Creative Commons licence, unless indicated otherwise in a credit line to the material. If material is not included in the article's Creative Commons licence and your intended use is not permitted by statutory regulation or exceeds the permitted use, you will need to obtain permission directly from the copyright holder. To view a copy of this licence, visit http://creativecommons.org/licenses/by/4.0/. 
cepts. Mathematical modeling is an effective technique that produces some of the best results for optimization and quantitative productivity (see $[19,20])$. The mathematical approach for enzymes' kinetics has been discussed in different articles, and also different mathematical techniques have been used for analytical solution of proposed models of chemical reactions (see [19, 21-25]). In this regard, Sharpe and Lotka in 1923 studied a kinematic enzyme in mathematical form for ecology and epidemiology. Along the same lines, L. Michaelis and M. Menton [23] proposed a mathematical model for a basic reaction of an enzyme given by

$$
S+E \rightleftharpoons{ }_{\eta_{2}}^{\eta_{1}}[S E] \rightarrow^{\eta_{3}} \mathcal{P}+E
$$

Studying the chemical reaction (1), one can infer that when enzyme $E$ of one molecule is combined with substrate $S$ of one molecule we will obtain a complex $S E$ of one molecule. Here $\eta_{1}$ denotes the rate of formation of enzyme, $\eta_{2}$ is the rate of creation of the product. Assume that the concentrations of $S, E, S E$ are equal to $\mathcal{S}, \mathcal{E}, \mathcal{C}$, respectively, i.e.,

$$
[S]=\mathcal{S}, \quad[E]=\mathcal{E}, \quad[S E]=\mathcal{C},
$$

where the concentration of a substance is represented by square brackets []. The author in [26] used the law of mass action to derive the following four nonlinear differential equations:

$$
\begin{aligned}
& \dot{\mathcal{S}}(\tau)=\eta_{2} \mathcal{C}-\eta_{1} \mathcal{E S} \\
& \dot{\mathcal{E}}(\tau)=\eta_{3} \mathcal{C}+\eta_{2} \mathcal{C}-\eta_{1} \mathcal{E S} \\
& \dot{\mathcal{C}}(\tau)=-\eta_{3} \mathcal{C}-\eta_{2} \mathcal{C}+\eta_{1} \mathcal{E} \mathcal{S} \\
& \dot{\mathcal{P}}(\tau)=\eta_{3} \mathcal{C} .
\end{aligned}
$$

In addition, one supplements the conditions $\mathcal{S}(0)=\mathcal{S}_{0}, \mathcal{C}(0)=0, \mathcal{E}(0)=\mathcal{E}_{0}$, and $\mathcal{P}(0)=0$. Here $\eta_{1}, \eta_{2}, \eta_{3}, \mathcal{S}_{0}$, and $\mathcal{E}_{0}$ are positive constants.

Alicea [24] solved the system of chemical reaction (2) by using multiple time scales method asymptotically. Alawneh [27] solved model (2) by a generalized differential transform method with multisteps and discussed the estimation analysis via fractional order derivative. Using the physical interpretation of the solution of system (2) with concentrations $\mathcal{S}, \mathcal{E}, \mathcal{C}$, and $\mathcal{P}$ at any time $\tau$ obtained mathematically, one observes that most of the time it does not coincide with the results obtained experimentally. In order to overcome such deficiency, one way is to use another type of differential operator instead of integer order derivative, which is usually known as fractional derivative. The researchers paid considerable attention to the aforementioned class of derivatives because they are more flexible and accurate compared with the classical derivatives, we refer the interested readers to $[4,28]$. Keeping these applications of fractional differential operator in mind, we introduce the following noninteger order enzyme kinematic system:

$$
\left\{\begin{array}{l}
{ }^{c} D^{\mu} \mathcal{S}(\tau)=\eta_{2} \mathcal{C}-\eta_{1} \mathcal{E S}, \\
{ }^{c} D^{\mu} \mathcal{C}(\tau)=-\eta_{3} \mathcal{C}-\eta_{2} \mathcal{C}+\eta_{1} \mathcal{E S}, \\
{ }^{c} D^{\mu} \mathcal{E}(\tau)=\eta_{3} \mathcal{C}+\eta_{2} \mathcal{C}-\eta_{1} \mathcal{E} \mathcal{S}, \\
{ }^{c} D^{\mu} \mathcal{P}(\tau)=\eta_{3} \mathcal{C},
\end{array}\right.
$$


subject to the initial conditions $\mathcal{S}(0)=\mathbf{n}_{1}, \mathcal{C}(0)=\mathbf{n}_{3}, \mathcal{E}(0)=\mathbf{n}_{2}, \mathcal{P}(0)=\mathbf{n}_{4}$, where ${ }^{c} D^{\mu}$ is noninteger order Caputo derivative and $0<\mu<1$. Further, $\mathbf{n}_{1}, \mathbf{n}_{2}>0$ and $\mathbf{n}_{3} \geq 0, \mathbf{n}_{4} \geq 0$. In the proposed system (3), the supplied conditions are independent of each other and also satisfy the relation $\mathcal{M}(\tau)=\mathcal{S}(\tau)+\mathcal{C}(\tau)+\mathcal{E}(\tau)+\mathcal{P}(\tau)$. The value of $M$ is used to represent terms presented in the concerned system of reactions.

Corresponding to model (3), we use the tools of fixed point theory to investigate some results that ensure the existence of the aforementioned model and its solution. We utilize Banach and Schauder's theorems from the proposed existence theory. We obtained the estimated solution of the model of noninteger order via Laplace transform combined with Adomian decomposition method which is known as Laplace Adomian Decomposition Method (LADM). LADM is an efficient technique by which we can find both explicit and analytic solutions for the system of differential equations. Those techniques are efficient and work outstandingly in both cases, i.e, in initial and boundary value problems. This method also works accurately for a system of stochastic differential equations. LADM does not need liberalization or perturbation like other existing computational and analytical schemes that need exploring the dynamical behavior of complex dynamical systems. The adopted techniques provide significant results for the solutions of FODEs, as well as for analytical solutions for the variety of problems of nonlinear differential equations.

In this paper, we utilize techniques of Adomian polynomials to decompose the nonlinearity and Laplace transform to convert the problem in hand into the form of algebraic equations, see [29]. Recently, the proposed techniques have been used to deal with nonsingular FODEs to obtain a very fruitful results (see [30]). To justify the results obtained via proposed techniques, we use Maple-13 and assign different values for the parameters and the supplied conditions. Furthermore, we remark that the obtained results via this method are in a form of convergent series, converging to the exact result uniformly. Thanks to the results of analysis obtained in [31-33], one can easily prove the convergence of the proposed method. Also, we construct the He homotopy perturbation method (HPM) to compute the concerned solutions for the proposed model. We compare both solutions and this HPM method worked very well (see [34, 35]).

\section{Preliminaries}

To make things easier, in this section we provide some well-known definitions, theorems, and lemmas. Their details are available in $[4,29,30]$.

Definition 1 The fractional integral in the sense of Riemann-Liouville of an arbitrary order $\mu$ for $f \in L^{1}([0, \infty), \mathbb{R})$ is given as

$$
I^{\mu} f(\tau)=\frac{1}{\Gamma(\mu)} \int_{0}^{\tau}(\tau-\varsigma)^{\mu-1} f(\varsigma) d \varsigma,
$$

such that the integral on the right-hand side is defined on $(0, \infty)$.

Definition 2 The Caputo fractional order derivative of a function $f$ is given by

$$
{ }^{c} D^{\mu} f(\tau)=\frac{1}{\Gamma(n-\mu)} \int_{0}^{\tau}(\tau-\varsigma)^{n-\mu-1} f^{(n)}(\varsigma) d \varsigma,
$$

the integral part of $\mu$ is represented by $[\mu]$ and $n=[\mu]+1$. The concerned derivative is used through this work. 
Lemma 1 In case of fractional differential equations, the following result holds:

$$
I^{\mu}\left[{ }^{c} D^{\mu} h\right](\tau)=h(\tau)+\xi_{0}+\xi_{1} \tau+\xi_{2} \tau^{2}+\cdots+\xi_{n-1} \tau^{n-1},
$$

where $\xi_{k}$ is any real number, $k$ is a positive integer up to $n-1$ and the integral part of $\mu$ is denoted by $[\mu]$, while $n=[\mu]+1$.

Definition 3 The Laplace transform in the sense of proposed derivative (Caputo) as defined by

$$
\mathcal{L}\left\{{ }^{c} D^{\mu} y(\tau)\right\}=s^{\mu} Y(s)-\sum_{j=0}^{n-1} s^{\mu-j-1} y^{(j)}(0), \quad n-1<\mu<n, n \in N .
$$

\section{Existence theory of enzyme kinetics model (3)}

This section is devoted to determining existence results for the proposed problem (3). In this connection, we define the following functions:

$$
\begin{aligned}
& \psi_{1}(\tau, \mathcal{S}, \mathcal{E}, \mathcal{C}, \mathcal{P})=\eta_{2} \mathcal{C}-\eta_{1} \mathcal{E S}, \\
& \psi_{2}(\tau, \mathcal{S}, \mathcal{E}, \mathcal{C}, \mathcal{P})=\eta_{3} \mathcal{C}+\eta_{2} \mathcal{C}-\eta_{1} \mathcal{E S}, \\
& \psi_{3}(\tau, \mathcal{S}, \mathcal{E}, \mathcal{C}, \mathcal{P})=-\eta_{3} \mathcal{C}-\eta_{2} \mathcal{C}+\eta_{1} \mathcal{E S}, \\
& \psi_{4}(\tau, \mathcal{S}, \mathcal{E}, \mathcal{C}, \mathcal{P})=\eta_{3} \mathcal{C} .
\end{aligned}
$$

Further, we set $\mathcal{B}=C[0, T]$ to be a Banach space with

$$
\|\mathbb{X}\|_{\mathcal{B}}=\sup _{\tau \in[0, T]}[|\mathcal{S}(\tau)|+|\mathcal{E}(\tau)|+|\mathcal{C}(\tau)|+|\mathcal{P}(\tau)|],
$$

where

$$
\mathbb{X}(\tau)=\left[\begin{array}{l}
\mathcal{S} \\
\mathcal{E} \\
\mathcal{C} \\
\mathcal{P}
\end{array}\right](\tau), \quad \mathbb{X}_{0}=\left[\begin{array}{c}
\mathcal{S}_{0} \\
\mathcal{E}_{0} \\
\mathcal{C}_{0} \\
\mathcal{P}_{0}
\end{array}\right](t), \quad \mathcal{H}(t, \mathbb{X}(t))=\left[\begin{array}{l}
\psi_{1}(\tau, \mathcal{S}, \mathcal{E}, \mathcal{C}, \mathcal{P}) \\
\psi_{2}(\tau, \mathcal{S}, \mathcal{E}, \mathcal{C}, \mathcal{P}) \\
\psi_{3}(\tau, \mathcal{S}, \mathcal{E}, \mathcal{C}, \mathcal{P}) \\
\psi_{4}(\tau, \mathcal{S}, \mathcal{E}, \mathcal{C}, \mathcal{P})
\end{array}\right](\tau)
$$

Keeping in mind the above notation, system (3) may be expressed as

$$
\begin{aligned}
& { }^{c} D^{\mu} \mathbb{X}(t)=\mathcal{H}(\tau, \mathbb{X}(\tau)), \quad \tau \in[0, T], \\
& \mathbb{X}(0)=\mathbb{X}_{0} .
\end{aligned}
$$

In view of $I^{\mu}$ on both sides of (5), we have

$$
\mathbb{X}(\tau)=\mathbb{X}_{0}+\int_{0}^{\tau} \frac{(\tau-y)^{\mu-1}}{\Gamma(\mu+1)} \mathcal{H}(y, \mathbb{X}(y)) d y, \quad \tau \in[0, T] .
$$

Let us define $\mathcal{A}: \mathcal{B} \rightarrow \mathcal{B}$ to be an operator by using (6) as

$$
\mathcal{A}(\mathbb{X})=\mathbb{X}, \quad \tau \in[0, T] .
$$


Further, we assume that the following hypothesis holds:

$\left(T_{1}\right)$ There exist constants $\mathbf{C}_{\mathcal{H}}, \mathbf{M}_{\mathcal{H}}$ with

$$
|\mathcal{H}(\tau, \mathbb{X}(\tau))| \leq \mathbf{C}_{\mathcal{H}}|\mathbb{X}(\tau)|+\mathbf{M}_{\mathcal{H}}, \quad \forall \mathbb{X} \in \mathcal{B}
$$

$\left(T_{2}\right)$ There exists a constant $\mathcal{L}_{\mathcal{H}}>0$ such that, for every $\mathbb{X}, \overline{\mathbb{X}} \in \mathcal{B}$,

$$
|\mathcal{H}(\tau, \mathbb{X})-\mathcal{H}(\tau, \overline{\mathbb{X}})| \leq \mathcal{L}_{\mathcal{H}}|\mathbb{X}-\overline{\mathbb{X}}|
$$

We established the results for at least one solution by utilizing Leray-Schauder fixed point theory.

Theorem 1 Under the continuity of $\mathcal{H}:[0, T] \times \mathcal{R}^{4} \rightarrow \mathcal{R}$ and assumption $\left(T_{1}\right)$, equation (7) has at least one fixed point. Consequently, the mathematical model (3) under consideration has at least one solution with $\omega \mathcal{C}_{\mathcal{H}}<1$, where $\omega=\frac{T^{\mu}}{\Gamma(\mu+1)}$.

Proof Let assumption $\left(T_{1}\right)$ hold. Define a set $\mathbf{D}=\left\{\mathbb{X}(\tau) \in \mathcal{B}:\|\mathbb{X}\|_{\mathcal{B}} \leq \rho, \tau \in[0, T]\right\}$, where $\rho \geq \max \frac{\sigma+\omega \mathcal{M}_{\mathcal{H}}}{1-\omega \mathcal{C}_{\mathcal{H}}}$. Clearly, $\mathbf{D}$ is a closed convex subset of $\mathcal{B}$. Now we prove that $\mathcal{A}: \mathbf{D} \rightarrow \mathbf{D}$. Letting $\mathbb{X} \in \mathbf{D}$, one has for $\left|\mathbb{X}_{0}\right|=\varpi, \omega=\frac{T^{\mu}}{\Gamma(\mu+1)}$,

$$
\begin{aligned}
|\mathcal{A} \mathbb{X}(\tau)| & =\left|\mathbb{X}_{0}+\int_{0}^{\tau} \frac{(\tau-y)^{\mu-1}}{\Gamma(\mu)} \mathcal{H}(y, \mathbb{X}(y)) d y\right| \\
& \leq \varpi+\int_{0}^{\tau} \frac{(\tau-y)^{\mu-1}}{\Gamma(\mu)}|\mathcal{H}(y, \mathbb{X}(y))| d y \\
& \leq \varpi+\omega \mathbf{C}_{\mathcal{H}} \rho+\omega \mathbf{M}_{\mathcal{H}} \\
& \leq \rho .
\end{aligned}
$$

This means that $\|\mathcal{A}(\mathbb{X})\|_{\mathcal{B}} \leq \rho$. Hence $\mathcal{A}(\mathbf{D}) \subset \mathbf{D}$. Also $\mathcal{A}$ is continuous.

Letting $\tau_{1}<\tau_{2} \in[0, T]$, we show that $\mathcal{A}$ is a completely continuous operator. For this, we have

$$
\begin{aligned}
\left|\mathcal{A X}\left(\tau_{2}\right)-\mathcal{A} \mathbb{X}\left(\tau_{1}\right)\right| & =\left|\int_{0}^{\tau_{2}} \frac{\left(\tau_{2}-y\right)^{\mu-1}}{\Gamma(\mu)} \mathcal{H}(y, \mathbb{X}(y)) d y-\int_{0}^{\tau_{1}} \frac{\left(\tau_{1}-y\right)^{\mu-1}}{\Gamma(\mu)} \mathcal{H}(y, \mathbb{X}(y)) d y\right| \\
& \leq \frac{\left(\mathbf{C}_{\mathcal{H}} \rho+\omega \mathbf{M}_{\mathcal{H}}\right)}{\Gamma(\mu+1)}\left[\tau_{2}^{\mu}-\tau_{1}^{\mu}\right]
\end{aligned}
$$

Now in (8), the right-hand side approaches zero when $\tau_{2} \rightarrow \tau_{1}$. Since $\mathcal{A}$ is bounded,

$$
\left\|\mathscr{A X}\left(\tau_{2}\right)-\mathcal{A} \mathbb{X}\left(\tau_{1}\right)\right\| \rightarrow 0, \quad \text { as } \tau_{2} \rightarrow \tau_{1}
$$

Thus by Arzelá-Ascoli theorem, $\mathcal{A}$ is relatively compact. Hence $\mathcal{A}$ is completely continuous. Thus via Schauder theorem, system (3) has a solution.

To ensure uniqueness of solution of (3), we have the next result.

Theorem 2 Under assumption ( $\left.T_{2}\right)$, system (3) has a unique solution if $T^{\mu} \mathbf{L}_{\mathcal{H}}<\Gamma(\mu+1)$. 
Proof Here we state that the first equation in this proof is given in (6). Further, if $\mathbb{X}, \overline{\mathbb{X}} \in \mathcal{B}$, $\mathcal{A}: \mathcal{B} \rightarrow \mathcal{B}$ is the operator defined above. Consider

$$
\begin{aligned}
\|\mathcal{A}(\mathbb{X})-\mathcal{A}(\overline{\mathbb{X}})\|_{\mathcal{B}} & =\max _{\tau \in[0, T]}\left|\int_{0}^{\tau} \frac{(\tau-y)^{\mu-1}}{\Gamma(\mu)} \mathcal{H}(y, \mathbb{X}(y)) d y-\int_{0}^{\tau} \frac{(\tau-y)^{\mu-1}}{\Gamma(\mu+1)} \mathcal{H}(y, \overline{\mathbb{X}}(y)) d y\right| \\
& \leq \max _{\tau \in[0, T]} \int_{0}^{\tau} \frac{(\tau-y)^{\mu-1}}{\Gamma(\mu)}|\mathcal{H}(y, \mathbb{X}(y))-\mathcal{H}(y, \overline{\mathbb{X}}(y))| d y \\
& \leq \frac{T^{\mu} \mathbf{L}_{\mathcal{H}}}{\Gamma(\mu+1)}\|\mathbb{X}-\overline{\mathbb{X}}\|_{\mathcal{B}}
\end{aligned}
$$

Hence, the operator $\mathcal{A}$ is a contraction. Thus the system has a unique solution.

\section{Construction of general solution via LADM}

In this segment, we discuss the general procedure for solving the proposed system of reactions (3) subject to the given conditions. Taking Laplace transform of the proposed model (3) yields

$$
\left\{\begin{array}{l}
\mathcal{L}\left\{{ }^{c} D^{\mu} \mathcal{S}(\tau)\right\}=\mathscr{L}\left\{-\eta_{1} \mathcal{E} \mathcal{S}+\eta_{2} \mathcal{C}\right\} \\
\mathcal{L}\left\{{ }^{c} D^{\mu} \mathcal{E}(\tau)\right\}=\mathscr{L}\left\{-\eta_{1} \mathcal{E} \mathcal{S}+\eta_{2} \mathcal{C}+\eta_{3} \mathcal{C}\right\} \\
\mathcal{L}\left\{{ }^{c} D^{\mu} \mathcal{C}(\tau)\right\}=\mathscr{L}\left\{\eta_{1} \mathcal{E S}-\eta_{2} \mathcal{C}-\eta_{3} \mathcal{C}\right\} \\
\mathcal{L}\left\{{ }^{c} D^{\mu} \mathcal{P}(\tau)\right\}=\mathcal{L}\left\{\eta_{3} \mathcal{C}\right\}
\end{array}\right.
$$

then, by applying the transformation on (10), we get

$$
\left\{\begin{array}{l}
s^{\mu} \mathcal{L}\{\mathcal{S}(\tau)\}-s^{\mu-1} \mathcal{S}(0)=\mathscr{L}\left\{-\eta_{1} \mathcal{E} \mathcal{S}+\eta_{2} \mathcal{C}\right\}, \\
s^{\mu} \mathcal{L}\{\mathcal{E}(\tau)\}-s^{\mu-1} \mathcal{E}(0)=\mathscr{L}\left\{-\eta_{1} \mathcal{E} \mathcal{S}+\eta_{2} \mathcal{C}+\eta_{3} \mathcal{C}\right\}, \\
s^{\mu} \mathcal{L}\{\mathcal{C}(\tau)\}-s^{\mu-1} \mathcal{C}(0)=\mathscr{L}\left\{\eta_{1} \mathcal{E} \mathcal{S}-\eta_{2} \mathcal{C}-\eta_{3} \mathcal{C}\right\}, \\
s^{\mu} \mathcal{L}\{\mathcal{P}(\tau)\}-s^{\mu-1} \mathcal{P}(0)=\mathscr{L}\left\{\eta_{3} \mathcal{C}\right\} .
\end{array}\right.
$$

Subject to initial conditions, equation (11) becomes

$$
\left\{\begin{array}{l}
\mathcal{L}[\mathcal{S}(\tau)]-\frac{\mathbf{n}_{1}}{s}=\frac{1}{s^{\mu}} \mathcal{L}\left\{-\eta_{1} \mathcal{E S}+\eta_{2} \mathcal{C}\right\} \\
\mathcal{L}[\mathcal{E}(\tau)]-\frac{\mathbf{n}_{2}}{s}=\frac{1}{s^{\mu}} \mathcal{L}\left\{-\eta_{1} \mathcal{E S}+\eta_{2} \mathcal{C}+\eta_{3} \mathcal{C}\right\} \\
\mathcal{L}[\mathcal{C}(\tau)]-\frac{\mathbf{n}_{3}}{s}=\frac{1}{s^{\mu}} \mathcal{L}\left\{\eta_{1} \mathcal{E} \mathcal{S}-\eta_{2} \mathcal{C}-\eta_{3} \mathcal{C}\right\} \\
\mathcal{L}[\mathcal{P}(\tau)]-\frac{\mathbf{n}_{4}}{s}=\frac{1}{s^{\mu}} \mathcal{L}\left\{\eta_{3} \mathcal{C}\right\}
\end{array}\right.
$$

We look for a solutions in the form of infinite series for $\mathcal{S}(\tau), \mathcal{E}(\tau), \mathcal{C}(\tau), \mathcal{P}(\tau)$ given as

$$
\begin{aligned}
& \mathcal{S}(\tau)=\sum_{n=0}^{\infty} \mathcal{S}_{n}(\tau), \quad \mathcal{E}(\tau)=\sum_{n=0}^{\infty} \mathcal{E}_{n}(\tau), \quad \mathcal{C}(\tau)=\sum_{n=0}^{\infty} \mathcal{C}_{n}(\tau) \\
& \mathcal{P}(\tau)=\sum_{n=0}^{\infty} \mathcal{P}_{n}(\tau) .
\end{aligned}
$$


We decompose the nonlinear term involved in the proposed model $\mathcal{E}(\tau) \mathcal{S}(\tau)$ by "Adomian polynomial" as

$$
\mathcal{E}(\tau) \mathcal{S}(\tau)=\sum_{n=0}^{\infty} A_{n}(\tau)
$$

where the "Adomian polynomials" $A_{n}$ are defined as

$$
A_{n}=\left.\frac{1}{n !}\left(\frac{d}{d \lambda}\right)^{n}\left[\sum_{k=0}^{n} \lambda^{k} \mathcal{E}_{k} \sum_{k=0}^{n} \lambda^{k} \mathcal{S}_{k}\right]\right|_{\lambda=0} \text {. }
$$

Using (13) and (14) in system (12), we obtain

$$
\left\{\begin{array}{l}
\mathcal{L}\left(\mathcal{S}_{0}\right)=\frac{\mathbf{n}_{1}}{s}, \quad \mathcal{L}\left(\mathcal{E}_{0}\right)=\frac{\mathbf{n}_{2}}{s}, \quad \mathcal{L}\left(\mathcal{C}_{0}\right)=\frac{\mathbf{n}_{3}}{s}, \quad \mathcal{L}\left(\mathcal{P}_{0}\right)=\frac{\mathbf{n}_{4}}{s}, \\
\mathcal{L}\left(\mathcal{S}_{1}\right)=\frac{1}{s^{\mu}} \mathcal{L}\left\{-\eta_{1} \mathcal{E}_{0} \mathcal{S}_{0}+\eta_{2} \mathcal{C}_{0}\right\}, \quad \mathcal{L}\left(\mathcal{E}_{1}\right)=\frac{1}{s^{\mu}} \mathcal{L}\left\{-\eta_{1} \mathcal{E}_{0} \mathcal{S}_{0}+\eta_{2} \mathcal{C}_{0}+\eta_{3} \mathcal{C}_{0}\right\}, \\
\mathcal{L}\left(\mathcal{C}_{1}\right)=\frac{1}{s^{\mu}} \mathcal{L}\left\{\eta_{1} \mathcal{E}_{0} \mathcal{S}_{0}-\eta_{2} \mathcal{C}_{0}-\eta_{3} \mathcal{C}_{0}\right\}, \quad \mathcal{L}\left(\mathcal{P}_{1}\right)=\frac{1}{s^{\mu}} \mathcal{L}\left\{\eta_{3} \mathcal{C}_{0}\right\}, \\
\mathcal{L}\left(\mathcal{S}_{2}\right)=\frac{1}{s^{\mu}} \mathcal{L}\left\{-\eta_{1} \mathcal{E}_{1} \mathcal{S}_{1}+\eta_{2} \mathcal{C}_{1}\right\}, \quad \mathcal{L}\left(\mathcal{E}_{2}\right)=\frac{1}{s^{\mu}} \mathcal{L}\left\{-\eta_{1} \mathcal{E}_{1} \mathcal{S}_{1}+\eta_{2} \mathcal{C}_{1}+\eta_{3} \mathcal{C}_{1}\right\}, \\
\mathcal{L}\left(\mathcal{C}_{2}\right)=\frac{1}{s^{\mu}} \mathcal{L}\left\{\eta_{1} \mathcal{E}_{1} \mathcal{S}_{1}-\eta_{2} \mathcal{C}_{1}-\eta_{3} \mathcal{C}_{1}\right\}, \quad \mathcal{L}\left(\mathcal{P}_{2}\right)=\frac{1}{s^{\mu}} \mathcal{L}\left\{\eta_{3} \mathcal{C}_{1}\right\}, \\
\vdots \\
\mathcal{L}\left(\mathcal{S}_{n+1}\right)=\frac{1}{s^{\mu}} \mathcal{L}\left\{-\eta_{1} \mathcal{E}_{n} \mathcal{S}_{n}+\eta_{2} \mathcal{C}_{n}\right\}, \quad \mathcal{L}\left(\mathcal{E}_{n+1}\right)=\frac{1}{s^{\mu}} \mathcal{L}\left\{-\eta_{1} \mathcal{E}_{n} \mathcal{S}_{n}+\eta_{2} \mathcal{C}_{n}+\eta_{3} \mathcal{C}_{n}\right\}, \\
\mathcal{L}\left(\mathcal{C}_{n+1}\right)=\frac{1}{s^{\mu}} \mathcal{L}\left\{\eta_{1} \mathcal{E}_{n} \mathcal{S}_{n}-\eta_{2} \mathcal{C}_{n}-\eta_{3} \mathcal{C}_{n}\right\}, \quad \mathcal{L}\left(\mathcal{P}_{n+1}\right)=\frac{1}{s^{\mu}} \mathcal{L}\left\{\eta_{3} \mathcal{C}_{n}\right\} .
\end{array}\right.
$$

Applying the inverse Laplace transform on (16), we have

$$
\begin{aligned}
\mathcal{S}_{0}= & \mathcal{L}^{-\mathscr{E}}\left(\frac{\mathbf{n}_{1}}{s}\right)=\mathbf{n}_{1}, \quad \mathcal{E}_{0}=\mathcal{L}^{-\mathscr{E}}\left(\frac{\mathbf{n}_{2}}{s}\right)=\mathbf{n}_{2}, \\
\mathcal{C}_{0}= & \mathcal{L}^{-\mathscr{E}}\left(\frac{\mathbf{n}_{3}}{s}\right)=\mathbf{n}_{3}, \quad \mathcal{P}_{0}=\mathcal{L}^{-\mathscr{E}}\left(\frac{\mathbf{n}_{4}}{s}\right)=\mathbf{n}_{4}, \\
\mathcal{S}_{1}= & \left(-\eta_{1} \mathbf{n}_{1} \mathbf{n}_{2}+\eta_{2} \mathbf{n}_{3}\right) \frac{\tau^{\mu}}{\Gamma(\mu+1)}, \quad \mathcal{E}_{1}=\left(-\eta_{1} \mathbf{n}_{1} \mathbf{n}_{2}+\eta_{2} \mathbf{n}_{3}+\eta_{3} \mathbf{n}_{3}\right) \frac{\tau^{\mu}}{\Gamma(\mu+1)}, \\
\mathcal{C}_{1}= & \left(\eta_{1} \mathbf{n}_{1} \mathbf{n}_{2}-\left(\eta_{2}+\eta_{3}\right) \mathbf{n}_{3}\right) \frac{\tau^{\mu}}{\Gamma(\mu+1)}, \quad \mathcal{P}_{1}=\left(\eta_{3} \mathbf{n}_{3}\right) \frac{\tau^{\mu}}{\Gamma(\mu+1)}, \\
\mathcal{S}_{2}= & -\eta_{1} \mathbf{n}_{2}\left(-\eta_{1} \mathbf{n}_{1} \mathbf{n}_{2}+\eta_{2} \mathbf{n}_{3}\right) \frac{\tau^{2 \mu}}{\Gamma(2 \mu+1)}-\eta_{1} \mathbf{n}_{1}\left(-\eta_{1} \mathbf{n}_{1} \mathbf{n}_{2}+\left(\eta_{2}+\eta_{3}\right) \mathbf{n}_{3}\right) \frac{\tau^{2 \mu}}{\Gamma(2 \mu+1)} \\
& +\eta_{2}\left(\eta_{1} \mathbf{n}_{1} \mathbf{n}_{2}-\left(\eta_{2}+\eta_{3}\right) \mathbf{n}_{3}\right) \frac{\tau^{2 \mu}}{\Gamma(2 \mu+1)}, \\
\mathcal{E}_{2}= & -\eta_{1} \mathbf{n}_{2}\left(-\eta_{1} \mathbf{n}_{1} \mathbf{n}_{2}+\eta_{2} \mathbf{n}_{3}\right) \frac{\tau^{2 \mu}}{\Gamma(2 \mu+1)}-\eta_{1} \mathbf{n}_{1}\left(-\eta_{1} \mathbf{n}_{1} \mathbf{n}_{2}+\left(\eta_{2}+\eta_{3}\right) \mathbf{n}_{3}\right) \frac{\tau^{2 \mu}}{\Gamma(2 \mu+1)} \\
& +\eta_{2}\left(\eta_{1} \mathbf{n}_{1} \mathbf{n}_{2}-\left(\eta_{2}+\eta_{3}\right) \mathbf{n}_{3}\right) \frac{\tau^{2 \mu}}{\Gamma(2 \mu+1)}+\eta_{3}\left(\eta_{1} \mathbf{n}_{1} \mathbf{n}_{2}-\left(\eta_{2}+\eta_{3}\right) \mathbf{n}_{3}\right) \frac{\tau^{2 \mu}}{\Gamma(2 \mu+1)}, \\
\mathcal{C}_{2}= & \eta_{1} \mathbf{n}_{2}\left(-\eta_{1} \mathbf{n}_{1} \mathbf{n}_{2}+\eta_{2} \mathbf{n}_{3}\right) \frac{\tau^{2 \mu}}{\Gamma(2 \mu+1)}+\eta_{1} \mathbf{n}_{1}\left(-\eta_{1} \mathbf{n}_{1} \mathbf{n}_{2}+\left(\eta_{2}+\eta_{3}\right) \mathbf{n}_{3}\right) \frac{\tau^{2 \mu}}{\Gamma(2 \mu+1)} \\
& -\eta_{2}\left(\eta_{1} \mathbf{n}_{1} \mathbf{n}_{2}-\left(\eta_{2}+\eta_{3}\right) \mathbf{n}_{3}\right) \frac{\tau^{2 \mu}}{\Gamma(2 \mu+1)}-\eta_{3}\left(\eta_{1} \mathbf{n}_{1} \mathbf{n}_{2}-\left(\eta_{2}+\eta_{3}\right) \mathbf{n}_{3}\right) \frac{\tau^{2 \mu}}{\Gamma(2 \mu+1)}
\end{aligned}
$$




$$
\mathcal{P}_{2}=\eta_{3}\left(\eta_{1} \mathbf{n}_{1} \mathbf{n}_{2}-\left(\eta_{2}+\eta_{3}\right) \mathbf{n}_{3}\right) \frac{\tau^{2 \mu}}{\Gamma(2 \mu+1)}
$$

In the same way, the other terms can be computed too. We assigned random values to $\mu$, in order to observe the mathematical dynamics of the above results $(\mathcal{S}(\tau), E(\tau), \mathcal{C}(\tau), p(\tau))$.

\section{Numerical results and discussion}

This section considers the semianalytic solution of the considered problem. We assigned the following values to the parameters and obtained estimated solution up to first four terms for the system:

$$
\begin{aligned}
& \mathbf{n}_{1}=10.00, \quad \mathbf{n}_{2}=01.00, \quad \mathbf{n}_{3}=\mathbf{n}_{4}=0 \\
& \eta_{1}=00.0730, \quad \eta_{2}=00.01, \quad \eta_{3}=00.030 \\
& \left\{\begin{array}{l}
\mathcal{S}_{0}=10.00, \quad \mathcal{E}_{0}=1.00, \quad \mathcal{C}_{0}=\mathcal{P}_{0}=0 \\
\mathcal{S}_{1}=\frac{-0.73}{\Gamma(\mu+1)} \tau^{\mu}, \quad \mathcal{E}_{1}=\frac{-0.73}{\Gamma(\mu+1)} \tau^{\mu}, \quad \mathcal{C}_{1}=\frac{0.73}{\Gamma(\mu+1)} \tau^{\mu}, \quad \mathcal{P}_{1}=0 \\
\mathcal{S}_{2}=\frac{0.59349}{\Gamma(2 \mu+1)} \tau^{2 \mu}, \quad \mathcal{E}_{2}=\frac{0.61539}{\Gamma(2 \mu+1)} \tau^{2 \mu} \\
\mathcal{C}_{2}=\frac{-0.61539}{\Gamma(2 \mu+1)} \tau^{2 \mu}, \quad \mathcal{P}_{2}=\frac{0.0219}{\Gamma(2 \mu+1)} \tau^{2 \mu} \\
\mathcal{S}_{3}=\frac{-0.8765}{\Gamma(3 \mu+1)} \tau^{3 \mu}+\frac{0.0686}{\Gamma(3 \mu+1)} \tau^{3 \mu} \\
\mathcal{E}_{3}=\frac{0.5986}{\Gamma(3 \mu+1)} \tau^{3 \mu}-\frac{0.7999}{\Gamma(3 \mu+1)} \tau^{3 \mu} \\
\mathcal{C}_{3}=\frac{0.7756}{\Gamma(3 \mu+1)} \tau^{3 \mu}-\frac{0.8820}{\Gamma(3 \mu+1)} \tau^{3 \mu} \\
\mathcal{P}_{3}=\frac{0.59349}{\Gamma(3 \mu+1)} \tau^{3 \mu}
\end{array}\right.
\end{aligned}
$$

Thus solutions with four terms become

$$
\left\{\begin{array}{l}
\mathcal{S}(\tau)=10-0.73 \frac{\tau^{\mu}}{\Gamma(\mu+1)}+0.593490 \frac{\tau^{2 \mu}}{\Gamma(2 \mu+1)}-0.8079 \frac{\tau^{3 \mu}}{\Gamma(3 \mu+1)}+0.0769 \frac{\tau^{4 \mu}}{\Gamma(4 \mu+1)}+\cdots \\
\mathcal{E}(\tau)=1-0.73 \frac{\tau^{\mu}}{\Gamma(\mu+1)}+0.61539 \frac{\tau^{2 \mu}}{\Gamma(2 \mu+1)}-0.2013 \frac{\tau^{3 \mu}}{\Gamma(3 \mu+1)}+0.7891 \frac{\tau^{4 \mu}}{\Gamma(4 \mu+1)}+\cdots \\
\mathcal{C}(\tau)=0.73 \frac{\tau^{\mu}}{\Gamma(\mu+1)}-0.61539 \frac{\tau^{2 \mu}}{\Gamma(2 \mu+1)}-0.1064 \frac{\tau^{3 \mu}}{\Gamma(3 \mu+1)}+0.7999 \frac{\tau^{4 \mu}}{\Gamma(4 \mu+1)}+\cdots \\
\mathcal{P}(\tau)=0.0219 \frac{\tau^{2 \mu}}{\Gamma(2 \mu+1)}+0.59349 \frac{\tau^{3 \mu}}{\Gamma(3 \mu+1)}+0.0054949 \frac{\tau^{4 \mu}}{\Gamma(4 \mu+1)}+\cdots
\end{array}\right.
$$

Now computing equation (19) for $\mu=0.9$, we have

$$
\left\{\begin{aligned}
\mathcal{S}(\tau)= & 10-0.7590205181 \tau^{0.9}+0.3540073134 \tau^{1.8} \\
& -0.1364823075 \tau^{2.7}+0.04805750170 \tau^{3.6}+\cdots \\
\mathcal{E}(\tau)= & 1-0.7590205181 \tau^{0.9}+0.3670703139 \tau^{1.8} \\
& +0.1409088818 \tau^{2.7}+0.04937504746 \tau^{3.6}+\cdots \\
\mathcal{C}(\tau)= & 0.7590205181 \tau^{0.9}-0.3670703139 \tau^{1.8} \\
& +0.1409088818 \tau^{2.7}-0.04937504746 \tau^{3.6}+\cdots \\
\mathcal{P}(\tau)= & 0.01306300049 \tau^{1.8} \\
& +0.004426574296 \tau^{2.7}+0.001317545754 \tau^{3.6}+\cdots
\end{aligned}\right.
$$


The approximate solution of equation (19) at $\mu=0.7$, is given by

$$
\left\{\begin{aligned}
\mathcal{S}(\tau)= & 10-0.8033996060 \tau^{0.7}+0.4777850967 \tau^{1.4} \\
& -0.2535660087 \tau^{2.1}+0.1261417992 \tau^{2.8}+\cdots, \\
\mathcal{E}(\tau)= & -0.8033996060 \tau^{0.7}+0.4954155426 \tau^{1.4} \\
& -0.2619667775 \tau^{2.1}+0.1298210630 \tau^{2.8}+\cdots, \\
\mathcal{C}(\tau)= & 0.8033996060 \tau^{0.7}-0.4954155426 \tau^{1.4} \\
& +0.2619667775 \tau^{2.1}-0.1298210630 \tau^{2.8}+\cdots \\
\mathcal{P}(\tau)= & 0.01763044637 \tau^{1.4} \\
& +0.00840076885 \tau^{2.1}+0.003679263766 \tau^{2.8}+\cdots
\end{aligned}\right.
$$

Similarly, for $\mu=0.5$, the approximate solution of equation (19) is

$$
\left\{\begin{aligned}
\mathcal{S}(\tau)= & 10-0.8237167919 \tau^{0.5}+0.5934900001 \tau \\
& -0.4124184879 \tau^{1.5}+0.2780081511 \tau^{2}+\cdots \\
\mathcal{E}(\tau)= & 1-0.8237167919 \tau^{0.5}+0.6153900001 \tau \\
& -0.4263063530 \tau^{1.5}+0.2865087449 \tau^{2}+\cdots \\
\mathcal{C}(\tau)= & 0.8237167919 \tau^{0.5}-0.6153900001 \tau \\
& +0.4263063530 \tau^{1.5}-0.2865087449 \tau^{2}+\cdots \\
\mathcal{P}(\tau)= & 0.02190000000 \tau \\
& +0.01388786511 \tau^{1.5}+0.008500593795 \tau^{2} \cdots
\end{aligned}\right.
$$

\section{Construction of general solution via HPM}

To illustrate the homotopy perturbation method (HPM) for solving the proposed model, according to $\mathrm{He}[36,37]$, the perturbation equations are defined from (3) as

$$
\left\{\begin{array}{l}
(1-\varepsilon)\left[{ }^{c} D^{\mu} \mathcal{S}(\tau)-{ }^{c} D^{\mu} \mathcal{S}_{0}(\tau)\right]+\varepsilon\left[{ }^{c} D^{\mu} \mathcal{S}(\tau)-\eta_{2} \mathcal{C}+\eta_{1} \mathcal{E S}\right]=0 \\
(1-\varepsilon)\left[{ }^{c} D^{\mu} \mathcal{C}(\tau)-{ }^{c} D^{\mu} \mathcal{C}_{0}(\tau)\right]+\varepsilon\left[{ }^{c} D^{\mu} \mathcal{C}(\tau)+\eta_{3} \mathcal{C}+\eta_{2} \mathcal{C}-\eta_{1} \mathcal{E}\right]=0 \\
(1-\varepsilon)\left[{ }^{c} D^{\mu} \mathcal{E}(\tau)-{ }^{c} D^{\mu} \mathcal{E}_{0}(\tau)\right]+\varepsilon\left[{ }^{c} D^{\mu} \mathcal{E}(\tau)+\eta_{3} \mathcal{C}-\eta_{2} \mathcal{C}+\eta_{1} \mathcal{E S}\right]=0 \\
(1-\varepsilon)\left[{ }^{c} D^{\mu} \mathcal{P}(\tau)-{ }^{c} D^{\mu} \mathcal{P}_{0}(\tau)\right]+\varepsilon\left[{ }^{c} D^{\mu} \mathcal{P}(\tau)-\eta_{3} \mathcal{C}\right]=0
\end{array}\right.
$$

where $\left(\mathcal{S}_{0}, \mathcal{C}_{0}, \mathcal{E}_{0}, \mathcal{P}_{0}\right)$ is the initial approximation of system (3) and $\varepsilon \in[0,1]$ is the embedding parameter. If $\varepsilon=0$ in (23), we have a system of FDEs as

$$
\left\{\begin{array}{l}
{ }^{c} D^{\mu} \mathcal{S}(\tau)-{ }^{c} D^{\mu} \mathcal{S}_{0}(\tau)=0 \\
{ }^{c} D^{\mu} \mathcal{C}(\tau)-{ }^{c} D^{\mu} \mathcal{C}_{0}(\tau)=0 \\
{ }^{c} D^{\mu} \mathcal{E}(\tau)-{ }^{c} D^{\mu} \mathcal{E}_{0}(\tau)=0 \\
{ }^{c} D^{\mu} \mathcal{P}(\tau)-{ }^{c} D^{\mu} \mathcal{P}_{0}(\tau)=0
\end{array}\right.
$$


whose solution is easy to determine. If $\varepsilon=1$ in (23), we get the original model (3). We may derive the required solution in the form of an infinite series for each compartment as

$$
\mathcal{S}=\sum_{n=0}^{\infty} \varepsilon^{n} \mathcal{S}_{n}, \quad \mathcal{C}=\sum_{n=0}^{\infty} \varepsilon^{n} \mathcal{C}_{n}, \quad \mathcal{E}=\sum_{n=0}^{\infty} \varepsilon^{n} \mathcal{E}_{n}, \quad \mathcal{P}=\sum_{n=0}^{\infty} \varepsilon^{n} \mathcal{P}_{n} .
$$

Therefore, the approximate solution of the original system (3) can be obtained by setting $\varepsilon=1$ in (25). Therefore, putting (25) into (23) and comparing the coefficients of terms with identical powers of $\varepsilon$ yields:

$$
\varepsilon^{0}:\left\{\begin{array}{l}
\mathcal{S}_{0}(\tau)=\mathbf{n}_{1} \\
\mathcal{C}_{0}(\tau)=\mathbf{n}_{3} \\
\mathcal{E}_{0}(\tau)=\mathbf{n}_{2} \\
\mathcal{P}_{0}(\tau)=\mathbf{n}_{4}
\end{array}\right.
$$

Similarly, one has

$$
\varepsilon^{1}:\left\{\begin{array}{l}
\mathcal{S}_{1}(\tau)=-\left(\eta_{1} \mathbf{n}_{1} \mathbf{n}_{2}-\eta_{2} \mathbf{n}_{3}\right) \frac{\tau^{\mu}}{\Gamma(\mu+1)}, \\
\mathcal{C}_{1}(\tau)=\left(\eta_{1} \mathbf{n}_{1} \mathbf{n}_{2}-\left(\eta_{2}+\eta_{3}\right) \mathbf{n}_{3}\right) \frac{\tau^{\mu}}{\Gamma(\mu+1)} \\
\mathcal{E}_{1}(\tau)=\left(-\eta_{1} \mathbf{n}_{1} \mathbf{n}_{2}+\eta_{2} \mathbf{n}_{3}+\eta_{3} \mathbf{n}_{3}\right) \frac{\tau^{\mu}}{\Gamma(\mu+1)} \\
\mathcal{P}_{1}(\tau)=\left(\eta_{3} \mathbf{n}_{3}\right) \frac{\tau^{\mu}}{\Gamma(\mu+1)}
\end{array}\right.
$$

and

$$
\varepsilon^{2}:\left\{\begin{aligned}
\mathcal{S}_{2}= & -\eta_{1} \mathbf{n}_{2}\left(-\eta_{1} \mathbf{n}_{1} \mathbf{n}_{2}+\eta_{2} \mathbf{n}_{3}\right) \frac{\tau^{2 \mu}}{\Gamma(2 \mu+1)}-\eta_{1} \mathbf{n}_{1}\left(-\eta_{1} \mathbf{n}_{1} \mathbf{n}_{2}+\left(\eta_{2}+\eta_{3}\right) \mathbf{n}_{3}\right) \frac{\tau^{2 \mu}}{\Gamma(2 \mu+1)} \\
& +\eta_{2}\left(\eta_{1} \mathbf{n}_{1} \mathbf{n}_{2}-\left(\eta_{2}+\eta_{3}\right) \mathbf{n}_{3}\right) \frac{\tau^{2 \mu}}{\Gamma(2 \mu+1)} \\
\mathcal{E}_{2}= & -\eta_{1} \mathbf{n}_{2}\left(-\eta_{1} \mathbf{n}_{1} \mathbf{n}_{2}+\eta_{2} \mathbf{n}_{3}\right) \frac{\tau^{2 \mu}}{\Gamma(2 \mu+1)}-\eta_{1} \mathbf{n}_{1}\left(-\eta_{1} \mathbf{n}_{1} \mathbf{n}_{2}+\left(\eta_{2}+\eta_{3}\right) \mathbf{n}_{3}\right) \frac{\tau^{2 \mu}}{\Gamma(2 \mu+1)} \\
& +\eta_{2}\left(\eta_{1} \mathbf{n}_{1} \mathbf{n}_{2}-\left(\eta_{2}+\eta_{3}\right) \mathbf{n}_{3}\right) \frac{\tau^{2 \mu}}{\Gamma(2 \mu+1)}+\eta_{3}\left(\eta_{1} \mathbf{n}_{1} \mathbf{n}_{2}-\left(\eta_{2}+\eta_{3}\right) \mathbf{n}_{3}\right) \frac{\tau^{2 \mu}}{\Gamma(2 \mu+1)} \\
\mathcal{C}_{2}= & \eta_{1} \mathbf{n}_{2}\left(-\eta_{1} \mathbf{n}_{1} \mathbf{n}_{2}+\eta_{2} \mathbf{n}_{3}\right) \frac{\tau^{2 \mu}}{\Gamma(2 \mu+1)}+\eta_{1} \mathbf{n}_{1}\left(-\eta_{1} \mathbf{n}_{1} \mathbf{n}_{2}+\left(\eta_{2}+\eta_{3}\right) \mathbf{n}_{3}\right) \frac{\tau^{2 \mu}}{\Gamma(2 \mu+1)} \\
& -\eta_{2}\left(\eta_{1} \mathbf{n}_{1} \mathbf{n}_{2}-\left(\eta_{2}+\eta_{3}\right) \mathbf{n}_{3}\right) \frac{\tau^{2 \mu}}{\Gamma(2 \mu+1)}-\eta_{3}\left(\eta_{1} \mathbf{n}_{1} \mathbf{n}_{2}-\left(\eta_{2}+\eta_{3}\right) \mathbf{n}_{3}\right) \frac{\tau^{2 \mu}}{\Gamma(2 \mu+1)} \\
\mathcal{P}_{2}= & \eta_{3}\left(\eta_{1} \mathbf{n}_{1} \mathbf{n}_{2}-\left(\eta_{2}+\eta_{3}\right) \mathbf{n}_{3}\right) \frac{\tau^{2 \mu}}{\Gamma(2 \mu+1)}
\end{aligned}\right.
$$

In this way, the higher order terms may be computed. Here we have obtained the same solution as obtained by LADM. Hence both methods can be used as a powerful tools to investigate nonlinear FDEs. Here, we plot the approximate solutions for the first three terms of both methods, LADM and HPM, via Matlab in Figs. 1, 2, 3, 4.

Figure 1 shows that the concentration of $\mathcal{S}$ is decreasing at different rates. For a smaller fractional order, this decrease is rapid as the order increasing the rate of decreasing is approaching the integer order. On the other hand, concentrations of the product substrates $\mathcal{C}, \mathcal{E}$, and $\mathcal{P}$ are increasing with a different rate as shown in Figs. 2, 3, 4, respectively. The increase rate is faster for a small fractional order. On the other hand, when enlarging the order, the process is becoming slow. So, a smaller fractional order model of enzymes will become more stable compared to larger order. Therefore, fractional order models will be more suitable for modeling the enzymes process instead of integer order. Also, for a 


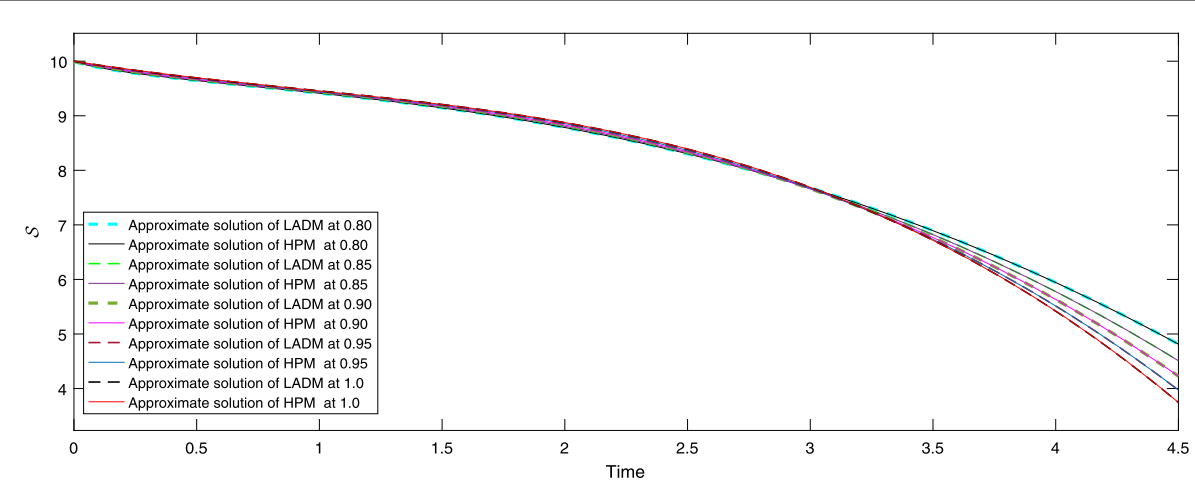

Figure 1 Comparison between the approximate solutions for $\mathcal{S}$ of model (3) for various fractional orders up to first three terms

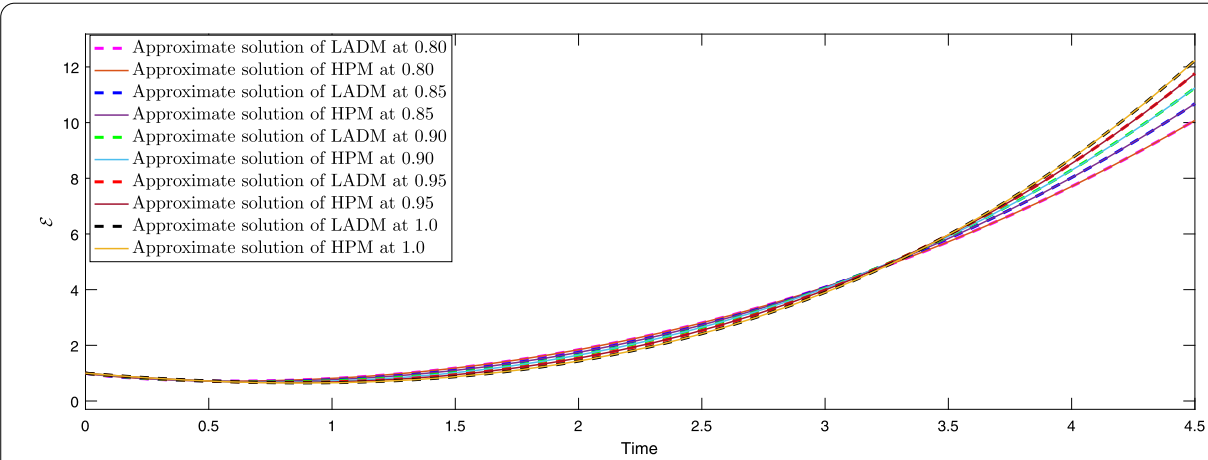

Figure 2 Comparison between the approximate solutions for $\mathcal{E}$ of model (3) for various fractional orders up to first three terms

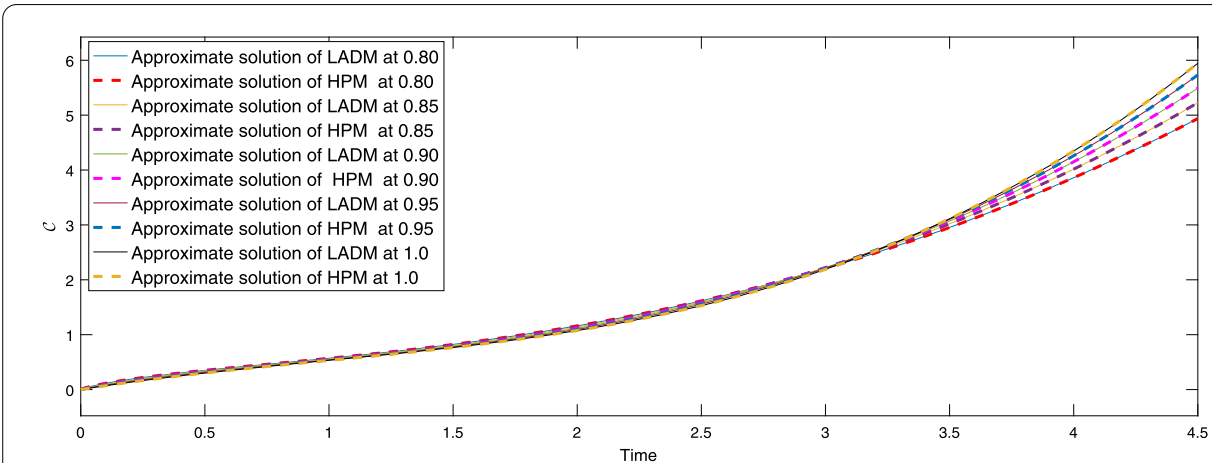

Figure 3 Comparison between the approximate solutions for $\mathcal{C}$ of model (3) for various fractional orders up to first three terms

smaller order, the reactants are more rapidly converging to the product. Both solutions of LADM and HPM are closely related with each other.

Remark 1 For the convergence of LADM, we refer to [38, Theorem 4.1], and for the convergence of HPM, see [39, Theorem 3.1]. 


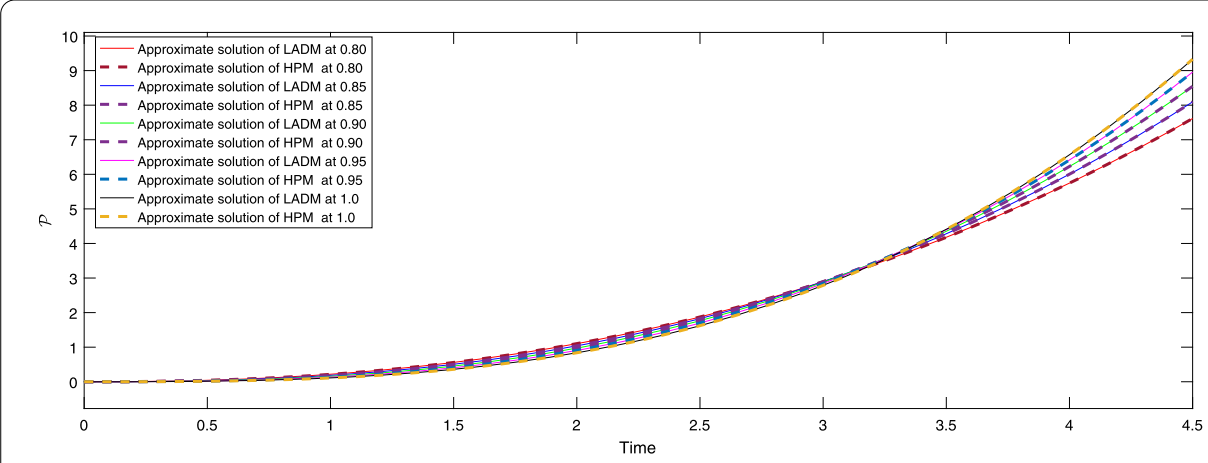

Figure 4 Comparison between the approximate solutions for $\mathcal{P}$ of model (3) for various fractional orders up to first three terms

\section{Conclusions}

The presented work is devoted to a fractional-order model of enzyme kinetics, which plays a role of a catalyst in various reactions taking place in living organisms. By means of nonlinear analysis, we have derived some results about the existence of at least one solution for the model under consideration. With the help of LADM, we have developed the semianalytic results for the proposed noninteger order model (3). The presented approximate results have been obtained by using famous He's HPM. Both methods have resulted the same solution. We have plotted the proposed solutions for both methods up to the first three terms, which completely agree with each other. Both methods can be used as a powerful mathematical tools to deal with many nonlinear problems of FDEs.

\section{Acknowledgements}

We are thankful to the reviewers for their careful reading and constructive suggestions which improved this paper considerably.

\section{Funding}

Not available.

Availability of data and materials

Data sharing not applicable to this article.

\section{Competing interests}

There does not exist any conflict of interest regarding this work.

\section{Authors' contributions}

$\mathrm{HA}$ and KS have given the formal analysis. AA and FH have written the paper and constructed the methodology. $\mathrm{HA}$ and KS have obtained the numerical results and summarized the discussion. All authors read and approved the final manuscript.

\section{Author details}

${ }^{1}$ College of Engineering, Al Ain University, Al Ain, United Arab Emirates. ${ }^{2}$ Department of Mathematics, Tafila Technical University, Tafila, Jordan. ${ }^{3}$ Department of Mathematics, Government Post Graduate Jahanzeb College, Saidu Sharif, 18000 Khyber Pakhtunkhwa, Pakistan. ${ }^{4}$ Department of Mathematics \& Statistics, University of Swat, Mingora, 18000 Khyber Pakhtunkhwa, Pakistan. ${ }^{5}$ Department of Mathematics, University of Malakand, Chakdara, 18000 Khyber Pakhtunkhwa, Pakistan.

\section{Publisher's Note}

Springer Nature remains neutral with regard to jurisdictional claims in published maps and institutional affiliations.

Received: 11 May 2020 Accepted: 13 August 2020 Published online: 27 August 2020

References

1. Hilfer, R.: Applications of Fractional Calculus in Physics. World Scientific, Singapore (2000) 
2. Kilbas, A.A., Marichev, O.I., Samko, S.G.: Fractional Integrals and Derivatives. Theory and Applications. Gordon \& Breach, Montreux (1993)

3. Kilbas, A.A., Srivastava, H.M., Trujillo, J.J.: Theory and Applications of Fractional Differential Equations. Elsevier, Amsterdam (2006)

4. Miller, K.S., Ross, B.: An Introduction to the Fractional Calculus and Fractional Differential Equations. Wiley, New York (1993)

5. Podlubny, l.: Fractional Differential Equations. Mathematics in Science and Engineering. Academic Press, New York (1999)

6. Rahimkhani, P., Ordokhani, Y., Babolian, E.: Numerical solution of fractional pantograph differential equations by using generalized fractional-order Bernoulli wavelet. J. Comput. Appl. Math. 309, 493-510 (2017)

7. Saeed, U., Rehman, M.U.: Hermite wavelet method for fractional delay differential equations. J. Differ. Equ. 2014 Article ID 359093 (2014)

8. Yang, Y., Huang, Y.: Spectral-collocation methods for fractional pantograph delay integrodifferential equations. Adv. Math. Phys. 2013, Article ID 821327 (2013)

9. Ishteva, M.K.: Properties and application of the Caputo fractional operator. Dept. Math., Univ. Karlsruhe (2005)

10. Zhou, Y:: Basic Theory of Fractional Differential Equations. World Scientific, Hackensack (1964)

11. Ali, A., Shah, K., Khan, R.A.: Existence of positive solution to a class of boundary value problems of fractional differential equations. Comput. Methods Differ. Equ. 4, 19-29 (2016)

12. Ali, A., Shah, K., Khan, R.A.: Existence and stability of solution to a toppled systems of differential equations of non-integer order. Bound. Value Probl. 2017, Article ID 16 (2017)

13. Caputo, M.: Linear models of dissipation whose $Q$ is almost frequency independent-II. Geophys. J. R. Astron. Soc. 13, 529-539 (1967)

14. Lakshmikantham, V., Leela, S., Vasundhara, J.: Theory of Fractional Dynamic Systems. Cambridge Academic Publishers, Cambridge (2009)

15. Cai, L., Wu, J:: Analysis of an HIV/AIDS treatment model with a nonlinear incidence rate. Chaos Solitons Fractals 41 , 175-182 (2009)

16. Wu, R.C., Hei, X.D., Chen, L.P.: Finite-time stability of fractional-order neural networks with delay. Commun. Theor. Phys. 60, 189-193 (2013)

17. Nanware, A., Dhaigude, D.B.: Existence and uniqueness of solutions of differential equations of fractional order with integral boundary conditions. J. Nonlinear Sci. Appl. 7, 246-254 (2014)

18. Agarwal, R.P., Belmekki, M., Benchohra, M.: A survey on semilinear differential equations and inclusions involving Riemann-Liouville fractional derivative. Adv. Differ. Equ. 2009, Article ID 981728 (2009)

19. Bonnans, J.F., Hermant, A.: Revisiting the analysis of optimal control problems with several state constraints. Control Cybern. 38, 1021-1052 (2009)

20. Wei, H.M., Li, X.Z., Martcheva, M.: An epidemic model of a vector-born disease with direct transmission and time delay. J. Math. Anal. Appl. 342, 895-908 (2008)

21. Lyons, M.E.G., Greer, J.C., Fitzgerald, C.A., Bannon, T., Barlett, P.N.: Reaction/diffusion with Michaelis-Menten kinetics in electroactive polymer films. Part 1. The steady-state amperometric response. Analyst 121,715-731 (1996)

22. Lyons, M.E.G., Bannon, T., Hinds, G., Rebouillat, S.: Reaction/diffusion with Michaelis-Menten kinetics in electroactive polymer films. Part 2. The transient amperometric response. Analyst 123, 1947-1959 (1998)

23. Michaelis, L., Menten, M.: Die Kinetik der Invertinwirkung. Biochem. Z. 49, 333-345 (1913)

24. Alicea, R.M.: A mathematical model for enzyme kinetics multiple time scale analysis. Dyn. Horsetooth 2A, 1-9 (2010)

25. Sharmila, D.C., Praveen, T., Rajendran, L.: Mathematical modeling and analysis of nonlinear enzyme catalyzed reaction processes. J. Theor. Chem. 2013, Article ID 931091 (2013)

26. Murray, J.D.: Mathematical Biology. Springer, Berlin (1989)

27. Alawneh, A.: Application of the multi-step generalized differential transform method to solve a time-fractional enzyme kinetics. Discrete Dyn. Nat. Soc. 2013, Article ID 592938 (2013)

28. Hu, Y., He, J.: On fractal space-time and fractional calculus. Therm. Sci. 3, 773-777 (2016)

29. Biazar, J.: Solution of the epidemic model by Adomian decomposition method. Appl. Math. Comput. 137, 1101-1106 (2006)

30. Shah, K., et al.: Semi-analytical study of pine wilt disease model with convex rate under Caputo-Fabrizio fractional order derivative. Chaos Solitons Fractals 135, Article ID 109754 (2020)

31. Abdilraze, A., Pelinosky, D.: Convergence of the Adomian decomposition method for initial value problems. Numer. Methods Partial Differ. Equ. 2009, 749-766 (2009)

32. Naghipour, A., Manafian, J.: Application of the Laplace Adomian decomposition method and implicit methods for solving Burger's equation. TWMS J. Pure Appl. Math. 6, 68-77 (2015)

33. Shah, K., Khalil, H., Khan, R.A.: Analytical solutions of fractional order diffusion equations by natural transform method. Iran. J. Sci. Technol. Trans. A, Sci. 42, 1479-1490 (2018)

34. Liao, S.J.: A kind of approximate solution technique which does not depend upon small parameters a special example. Int J. Non-Linear Mech. 30, 371-380 (1995)

35. Liao, S.J.: Introduction to the Homotopy Analysis Method. Chapman \& Hall/CRC Press, Boca Raton (2003)

36. He, J.H.: The homotopy perturbation method for nonlinear oscillators with discontinuities. Appl. Math. Comput. 151, 287-292 (2004)

37. Liu, Y., Li, Z., Zhang, Y:: Homotopy perturbation method to fractional biological population equation. Fract. Differ. Calc. 1(1), 117-124 (2011)

38. Shah, K., Bushnaq, S.: Numerical treatment of fractional endemic disease model via Laplace Adomian decomposition method. J. Sci. Arts 17(2), 257-268 (2017)

39. Ayati, Z., Biazar, J.: On the convergence of homotopy perturbation method. J. Egypt. Math. Soc. 23(2), 424-428 (2015) 